Pacific

Journal of

Mathematics

THE HEAT KERNEL AND THE RIESZ TRANSFORMS ON THE QUATERNIONIC HEISENBERG GROUPS

FuLIU ZhU 


\title{
THE HEAT KERNEL AND THE RIESZ TRANSFORMS ON THE QUATERNIONIC HEISENBERG GROUPS
}

\author{
Fuliu Zhu
}

\begin{abstract}
In this paper we use the method of stochastic integral due to Gaveau to construct the heat kernel for the quaternionic Heisenberg groups, and then follow the line of Coulhon et al. to deduce the uniformly boundedness of the Riesz transforms on these nilponent Lie groups.
\end{abstract}

\section{Introduction.}

As the heat kernel plays an important role in many problems in harmonic analysis, an explicit usable expression is very much desirable.

An explicit expression for the heat kernel for the Heisenberg group $H^{n}=$ $\mathbf{C}^{n} \times \mathbf{R}$ was obtained by Hulanicki [9] and by Gaveau [7]. Gaveau [7] also obtained the heat kernel for free nilpotent Lie groups of step two. Cygan [4] obtained the heat kernel for all nilpotent Lie groups of step two. But neither Gaveau's expression for free nilpotent Lie groups nor Cygan's expression for arbitrary nilpotent Lie groups of step two were as explicit as in the case of Heisenberg groups.

The Hulanicki-Gaveau's formula for the heat kernel for the Heisenberg group has many interesting applications: Hueber [8] et al. used it to describe the Martin boundary corresponding to the sublaplacian of the Heisenberg group, Garofalo [6] et al. used it to study the regularity of boundary points in the Dirichlet problem for the heat equation on the Heisenberg group, while Coulhon [3] et al. used it to show the uniform boundedness of Riesz transforms on the Heisenberg group. Although these applications are very impressive, they depend heavily on explicit expressions for the heat kernel. All of these works motivate the following question: Are there other nilpotent Lie groups for which the expressions for the heat kernel are as explicit as in the case of the Heisenberg group?

The first aim of this paper is to look for such formulae for the heat kernel for the quaternionic Heisenberg groups. These groups are defined by replacing the complex field $\mathbf{C}$ by the field of quaternions $\mathbf{H}$ in the definition of $\mathbf{H}^{n}$. More precisely, we make $\mathbf{H}^{n} \times \mathbf{R}^{3}$ into a nilpotent Lie group of step two by suitably defining the group operation. On this group there is a natural sublaplacian with an associated heat kernel. We use the method of Gaveau 
[7], i.e., the stochastic integral, to calculate the heat kernel for the quaternionic Heisenberg group and obtain a closed form expression which closely resembles that of the heat kernel for Heisenberg groups. As we know, apart from the standard Heisenberg group, the quaternionic Heisenberg group is the only nilpotent Lie group on which an explicit formula for the heat kernel has been obtained up to now.

The second aim of this paper is to use the explicit formula for the heat kernel to study the uniform boundedness of the Riesz transforms on the quaternionic Heisenberg group. That is, the Riesz transforms are bounded on $L^{p}$ spaces with norms independent of the dimension of the group. On the standard Heisenberg group this problem was addressed by Colhon [3] et al.. We apply their method to the quaternionic groups and by overcoming considerable difficulties in the process of calculation, finally prove the uniform boundedness of Riesz transforms on the quaternionic Heisenberg group.

We hope that we can use this explicit expression of the heat kernel to solve other problems in the harmonic analysis on the quaternionic Heisenberg group.

\section{Prelimilaries.}

We identify the division ring $\mathbf{H}$ of quaternions with $\mathbf{R} \times \mathbf{R}^{3}$. For $\mathbf{p}=$ $\left(x_{0}, \mathbf{x}\right), \mathbf{q}=\left(y_{0}, \mathbf{y}\right) \in \mathbf{H}$, the quaternionic multiplication is defined as:

$$
\mathbf{p q}=\left(x_{0}, \mathbf{x}\right)\left(y_{0}, \mathbf{y}\right)=\left(x_{0} y_{0}-\mathbf{x} \cdot \mathbf{y}, x_{0} \mathbf{y}+y_{0} \mathbf{x}+\mathbf{x} \times \mathbf{y}\right),
$$

where $\mathbf{x} . \mathbf{y}$ and $\mathbf{x} \times \mathbf{y}$ are the inner and exterior product of $\mathbf{x}$ and $\mathbf{y}$ respectively. For $\mathbf{p}=\left(x_{0}, \mathbf{x}\right) \in \mathbf{H}$, we use the notations $x_{0}=\operatorname{Re} \mathbf{p}, \mathbf{x}=\operatorname{Im} \mathbf{p}$. The conjugate of $\mathbf{p}$ is denoted as $\overline{\mathbf{p}}=\left(x_{0},-\mathbf{x}\right)$ and $|\mathbf{p}|=(\mathbf{p} \cdot \overline{\mathbf{p}})^{1 / 2}$ is the norm of p.

The product space $\mathbf{H}^{n} \times \mathbf{R}^{3}$ together with the multiplication

$$
\begin{aligned}
& \left(\mathbf{p}_{\mathbf{1}}, \ldots, \mathbf{p}_{\mathbf{n}}, \mathbf{u}\right) \cdot\left(\mathbf{q}_{\mathbf{1}}, \ldots, \mathbf{q}_{\mathbf{n}}, \mathbf{v}\right) \\
& =\left(\mathbf{p}_{\mathbf{1}}+\mathbf{q}_{\mathbf{1}}, \ldots, \mathbf{p}_{\mathbf{n}}+\mathbf{q}_{\mathbf{n}}, \mathbf{u}+\mathbf{v}+2 \sum_{r=1}^{n} \operatorname{Im}\left(\mathbf{q}_{r} \cdot \overline{\mathbf{p}}_{r}\right)\right)
\end{aligned}
$$

constitutes a Lie group, called the quaternionic Heisenberg group, and denoted by $\mathbf{H} H^{n}$ (Allcock's notation [1] ).

We know [2] that if

$$
\begin{aligned}
& \mathbf{p}_{r}=\left(x_{r 0}, \mathbf{x}_{\mathbf{r}}\right)=\left(x_{r 0},\left(x_{r 1}, x_{r 2}, x_{r 3}\right)\right) \\
& \text { for } 1 \leq r \leq n \text { and } \mathbf{u}=\left(u_{1}, u_{2}, u_{3}\right) \in \mathbf{R}^{3},
\end{aligned}
$$


then the vector fields

$$
\begin{aligned}
& X_{r 0}=\frac{\partial}{\partial x_{r 0}}-2 x_{r 1} \frac{\partial}{\partial u_{1}}-2 x_{r 2} \frac{\partial}{\partial u_{2}}-2 x_{r 3} \frac{\partial}{\partial u_{3}}, \\
& X_{r 1}=\frac{\partial}{\partial x_{r 1}}+2 x_{r 0} \frac{\partial}{\partial u_{1}}+2 x_{r 3} \frac{\partial}{\partial u_{2}}-2 x_{r 2} \frac{\partial}{\partial u_{3}} \\
& X_{r 2}=\frac{\partial}{\partial x_{r 2}}-2 x_{r 3} \frac{\partial}{\partial u_{1}}+2 x_{r 0} \frac{\partial}{\partial u_{2}}+2 x_{r 1} \frac{\partial}{\partial u_{3}} \\
& X_{r 3}=\frac{\partial}{\partial x_{r 3}}+2 x_{r 2} \frac{\partial}{\partial u_{1}}-2 x_{r 1} \frac{\partial}{\partial u_{2}}+2 x_{r 0} \frac{\partial}{\partial u_{3}}
\end{aligned}
$$

form a basis of the Lie algebra of $\mathbf{H} H^{n}$. The commutators of these vector fields satisfy

$$
\begin{aligned}
& {\left[X_{r 0}, X_{s 1}\right]=4 \delta_{r s} \frac{\partial}{\partial u_{1}}=\left[X_{r 2}, X_{s 3}\right],} \\
& {\left[X_{r 0}, X_{s 2}\right]=4 \delta_{r s} \frac{\partial}{\partial u_{2}}=\left[X_{r 3}, X_{s 1}\right],} \\
& {\left[X_{r 0}, X_{s 3}\right]=4 \delta_{r s} \frac{\partial}{\partial u_{3}}=\left[X_{r 1}, X_{s 2}\right]}
\end{aligned}
$$

with all other brackets equal to zero. So the quaternionic Heisenberg group is a nilpotent Lie group of step two.

Following the case of the Heisenberg groups [5], we introduce on $\mathbf{H} H^{n}$ the group $\left\{\delta_{t}: 0<t<\infty\right\}$ of dilations defined by

$$
\delta_{t}(\mathbf{p}, \mathbf{u})=\left(t \mathbf{p}, t^{2} \mathbf{u}\right)=\left(t \mathbf{p}_{1}, \ldots, t \mathbf{p}_{n}, t^{2} \mathbf{u}\right) .
$$

These dilations satisfy the distributive law

$$
\delta_{t}((\mathbf{p}, \mathbf{u}) \cdot(\mathbf{q}, \mathbf{v}))=\delta_{t}(\mathbf{p}, \mathbf{u}) \cdot \delta_{t}(\mathbf{q}, \mathbf{v}) .
$$

We also define the norm function on $\mathbf{H} H^{n}$ by

$$
|(\mathbf{p}, \mathbf{u})|=\left(|\mathbf{p}|^{4}+|\mathbf{u}|^{2}\right)^{1 / 4}=\left(\left(\sum_{r=1}^{n} \sum_{i=0}^{3}\left|x_{r i}\right|^{2}\right)^{2}+\sum_{j=1}^{3} u_{j}^{2}\right)^{1 / 4}
$$

which satisfies

$$
\left|\delta_{t}(\mathbf{p}, \mathbf{u})\right|=t|(\mathbf{p}, \mathbf{u})| .
$$

Let $e=(0,0)$ be the identity element of the group $\mathbf{H} H^{n}$.

We know [5] that Kohn's sublaplace operator on the quaternionic Heisenberg group is defined as

$$
\triangle=\sum_{r=1}^{n} \sum_{i=0}^{3} X_{r i}^{2}
$$


A simple calculation shows that

$$
\begin{aligned}
\triangle= & \sum_{r=1}^{n} \sum_{i=0}^{3} \frac{\partial^{2}}{\partial x_{r i}^{2}}+4 \sum_{r=1}^{n} \sum_{i=1}^{3}\left(x_{r 0} \frac{\partial^{2}}{\partial x_{r i} \partial u_{i}}-x_{r i} \frac{\partial^{2}}{\partial x_{r 0} \partial u_{i}}\right) \\
& +4 \sum_{r=1}^{n} \sum_{i=0}^{3} x_{r i}^{2} \sum_{j=1}^{3} \frac{\partial^{2}}{\partial u_{j}^{2}}+4 \sum_{r=1}^{n} \sum_{(i, j, k)} x_{r i}\left(\frac{\partial^{2}}{\partial x_{r j} \partial u_{k}}-\frac{\partial^{2}}{\partial x_{r k} \partial u_{j}}\right),
\end{aligned}
$$

where $(i, j, k)$ means the cyclic permutation of $(1,2,3)$.

\section{The heat kernels of the quaternionic Heisenberg groups.}

In this section we shall use the method developed by Gaveau [7] and Hulanicki [9] to derive an explicit expression of the heat kernel of the quaternionic Heisenberg group. Firstly we have:

Lemma 2.1. The diffusion of the infinitesimal generator $\frac{1}{2} \triangle$ starting at $e$ is the process

$$
g(s)=\left(x_{r i}(s), u_{j}(s)\right)_{1 \leq r \leq n, 0 \leq i \leq 3,1 \leq j \leq 3},
$$

where $\left(x_{r i}(s)\right)_{1 \leq r \leq n, 0 \leq i \leq 3}$ are $4 n$ standard Brownian motions, and

$$
u_{j}(s)=2 \sum_{r=1}^{n} \int_{0}^{s} x_{r 0} d x_{r j}(t)-x_{r j} d x_{r 0}(t)+x_{r k} d x_{r i}(t)-x_{r i} d x_{r k}(t) .
$$

Proof. As in [7], the projection on $\mathbf{H}^{n}=\mathbf{R}^{4 n}$ of the diffusion to be found is the diffusion of the infinitesimal generator $\frac{1}{2} \sum_{r=1}^{n} \sum_{i=0}^{3} \frac{\partial^{2}}{\partial x_{r i}{ }^{2}}$, which is given by $4 n$ standard Brownian motions $\left(\mathbf{x}_{1}(s), \ldots, \mathbf{x}_{n}(s)\right)$. Hence it is sufficient to compute the stochastic differentials $d u_{j}(1 \leq j \leq 3)$.

We observe that the matrix of principal symbols of $\triangle$ is given by

$$
G=\left(\begin{array}{cccc}
I_{4} & \ldots & 0 & A_{1} \\
\vdots & \ddots & \vdots & \vdots \\
0 & \ldots & I_{4} & A_{n} \\
{ }^{t} A_{1} & \ldots & { }^{t} A_{n} & C
\end{array}\right)
$$

where, for $1 \leq r \leq n$,

$$
A_{r}=\left(\begin{array}{ccc}
-2 x_{r 1} & -2 x_{r 2} & -2 x_{r 3} \\
2 x_{r 0} & 2 x_{r 3} & -2 x_{r 2} \\
-2 x_{r 3} & 2 x_{r 0} & 2 x_{r 1} \\
2 x_{r 2} & -2 x_{r 1} & 2 x_{r 0}
\end{array}\right)
$$

and

$$
C=\left(4 \sum_{r=1}^{n}\left|\mathbf{x}_{\mathbf{r}}\right|^{2}\right) I_{3}
$$


In these expressions, $I_{k}$ denotes the identity matrix of order $k$. Let

$$
\Sigma=\left(\begin{array}{cccc}
I_{4} & \cdots & 0 & A_{1} \\
\vdots & \ddots & \vdots & \vdots \\
0 & \cdots & I_{4} & A_{n}
\end{array}\right)
$$

It is obvious that

$$
{ }^{t} \Sigma \Sigma=G
$$

and we know that [7] the matrix $\Sigma$ gives stochastic differentials of the diffusion $\frac{1}{2} \triangle$ :

$$
\left(d \mathbf{x}_{1}, \ldots, d \mathbf{x}_{n}, d u_{1}, d u_{2}, d u_{3}\right)=\left(d \mathbf{x}_{1}, \ldots, d \mathbf{x}_{n}\right)\left(\begin{array}{cccc}
I_{4} & \ldots & 0 & A_{1} \\
\vdots & \ddots & \vdots & \vdots \\
0 & \ldots & I_{4} & A_{n}
\end{array}\right) .
$$

It is easy to obtain

$$
d u_{i}=2 \sum_{r=1}^{n}\left(x_{r 0} d x_{r i}-x_{r i} d x_{r 0}+x_{r j} d x_{r k}-x_{r k} d x_{r j}\right) .
$$

Let

$$
\frac{1}{2} \triangle=\frac{\partial}{\partial s}
$$

be the equation of propagation of heat, where $s(\geq 0)$ denotes the time. Assume that $p_{s}(e, \mathbf{g})$, for $\mathbf{g} \in \mathbf{H} H^{n}$, be the heat kernel with pole at $e$. From the definition we have

$$
p_{s}(e, \mathbf{g}) d \mathbf{g}=\operatorname{Prob}\left(\mathbf{g}_{\omega}(s) \in d \mathbf{g}\right),
$$

where $d \mathbf{g}$ is the left-invariant measure on $\mathbf{H} H^{n}$. Let

$$
\mathbf{y}=\left(\mathbf{y}_{1}, \ldots, \mathbf{y}_{n}\right)=\left(y_{10}, \ldots, y_{13}, \ldots, y_{n 0}, \ldots, y_{n 3}\right)
$$

and $\mathbf{v}=\left(v_{1}, v_{2}, v_{3}\right)$ be the dual variables of $\mathbf{x}=\left(\mathbf{x}_{1}, \ldots, \mathbf{x}_{n}\right)$ and $\mathbf{u}=$ $\left(u_{1}, u_{2}, u_{3}\right)$ respectively. We write $|\mathbf{x}|^{2}=\sum_{r=1}^{n} \sum_{i=0}^{3} x_{r i}^{2}$ and

$$
\begin{aligned}
a & =4\left(\begin{array}{cccc}
0 & v_{1} & v_{2} & v_{3} \\
-v_{1} & 0 & v_{3} & -v_{2} \\
-v_{2} & -v_{3} & 0 & v_{1} \\
-v_{3} & v_{2} & -v_{1} & 0
\end{array}\right), \\
A & =\operatorname{diag}(\underbrace{a, \ldots, a}_{n}) .
\end{aligned}
$$

If $X$ is a skew-symmetric matrix of order $4 n$ and $\mathbf{w} \in \mathbf{R}^{4 n}$, we write $\psi_{s}(X, \mathbf{w})=\exp \left[\frac{1}{s}\left(-|\mathbf{w}|^{2}+{ }^{t} \mathbf{w}\left(I_{4 n}-\frac{s^{2} X^{2}}{4 \pi^{2}}\right)^{-1} \mathbf{w}\right)\right] \operatorname{det}\left(I_{4 n}-\frac{s X}{2 \pi}\right)^{-1}$ 
where ${ }^{t} \mathbf{w}$ is the transpose of the vector $\mathbf{w}$.

With these preparations, we have:

Theorem 2.2. The Euclidean Fourier transform of $p_{s}(e, \mathbf{g})$ is given by the formula

$$
\begin{aligned}
& \hat{p}_{s}(e, .)(\mathbf{y}, \mathbf{v}) \\
& =(2 \pi s)^{-2 n} \int_{\mathbf{R}^{4 n}} \exp \left(\sqrt{-1} \sum_{r=1}^{n} \sum_{i=0}^{3} y_{r i} x_{r i}-\frac{|\mathbf{x}|^{2}}{2 s}\right) \prod_{m=1}^{\infty} \psi_{s}\left(\frac{A}{m}, \mathbf{x}\right) d \mathbf{x} .
\end{aligned}
$$

Proof. By the definition, we have

$$
\begin{aligned}
& \hat{p}_{s}(e, .)(\mathbf{y}, \mathbf{v}) \\
& =\int_{\mathbf{R}^{4 n+3}} \exp \left(\sqrt{-1}\left(\sum_{r=1}^{n} \sum_{i=0}^{3} y_{r i} x_{r i}+\sum_{j=1}^{3} v_{j} u_{j}\right)\right) p_{s}(e,(\mathbf{x}, \mathbf{u})) d \mathbf{x} d \mathbf{u} \\
& =E_{0}\left(\exp \sqrt{-1}\left(\sum_{r=0}^{n} \sum_{i=0}^{3} y_{r i} x_{r i}(s)+\sum_{j=1}^{3} v_{j} u_{j}(s)\right)\right) \\
& =E_{0}\left(\exp \sqrt{-1} \sum_{r=1}^{n} \sum_{i=0}^{3} y_{r i} x_{r i}(s)\right) \\
& \cdot E_{0}\left(\exp \sqrt{-1} \sum_{j=1}^{3} v_{j} u_{j}(s) \mid, x_{r i}(s)=x_{r i}\right),
\end{aligned}
$$

where $E_{0}\left(\ldots \mid x_{r i}(s)=x_{r i}\right)$ denotes the conditional expectation given the $x_{r i}(s)$. So it is sufficient to evaluate

$$
E_{0}\left(\exp \left(\sqrt{-1} \sum_{j=1}^{3} v_{j} u_{j}(s)\right) \mid x_{r i}(s)=x_{r i}\right) .
$$

We express the $4 n$ standard real Brownian motions as the Fourier series with independent Gaussian variables as their coefficients, i.e., when $s \neq 2 \pi$,

$$
x_{r i}(s)=\frac{s \xi^{(r i)}}{\sqrt{2 \pi}}+\sum_{m=1}^{\infty} \frac{1}{m \sqrt{\pi}}\left(\xi_{m}^{(r i)}(\cos m s-1)-\xi_{m}^{\prime(r i)} \sin m s\right),
$$

where $\xi_{m}^{(r i)}$ and $\xi_{m}^{\prime(r i)}$ are one-dimensional standard normal distributions which are independent of each other, and $x_{r i}(2 \pi)=U_{r i}$. 
From Lemma 2.1 we get immediately

$$
\begin{aligned}
u_{i}(2 \pi)= & 4 \sum_{r=1}^{n} \frac{1}{m}\left[\xi_{m}^{(r 0)}\left(\xi_{m}^{\prime(r i)}-\frac{U_{r i}}{\sqrt{\pi}}\right)-\xi_{m}^{(r i)}\left(\xi_{m}^{\prime(r 0)}-\frac{U_{r 0}}{\sqrt{\pi}}\right)\right. \\
& \left.+\xi_{m}^{(r j)}\left(\xi_{m}^{\prime(r k)}-\frac{U_{r k}}{\sqrt{\pi}}\right)-\xi_{m}^{(r k)}\left(\xi_{m}^{\prime(r j)}-\frac{U_{r j}}{\sqrt{\pi}}\right)\right] .
\end{aligned}
$$

Thus

$$
\begin{aligned}
& E_{0}\left(\exp \left(\sqrt{-1} \sum_{j=1}^{3} v_{j} u_{j}(2 \pi)\right) \mid x_{r i}(2 \pi)=U_{r i}\right) \\
& =\prod_{m=1}^{\infty} E_{0}\left(\operatorname { e x p } \frac { 4 \sqrt { - 1 } } { m } \left\{\sum _ { i = 1 } ^ { 3 } v _ { i } \sum _ { r = 1 } ^ { n } \left[\xi_{m}^{(r 0)}\left(\xi_{m}^{\prime}(r i)-\frac{U_{r i}}{\sqrt{\pi}}\right)\right.\right.\right. \\
& \quad-\xi_{m}^{(r i)}\left(\xi_{m}^{\prime(r 0)}-\frac{U_{r 0}}{\sqrt{\pi}}\right)+\xi_{m}^{(r j)}\left(\xi_{m}^{\prime(r k)}-\frac{U_{r k}}{\sqrt{\pi}}\right) \\
& \left.\left.\left.\quad-\xi_{m}^{(r k)}\left(\xi_{m}^{(r j)}-\frac{U_{r j}}{\sqrt{\pi}}\right)\right]\right\} \mid x_{r i}(2 \pi)=U_{r i}\right) .
\end{aligned}
$$

In the last expression, all conditional information is exhausted, so the conditional expectation is actually reduced to the expectation. Hence it is sufficient to consider the terms

$$
\begin{aligned}
J_{m}= & E\left(\operatorname { e x p } \frac { 4 \sqrt { - 1 } } { m } \left\{\sum _ { i = 1 } ^ { 3 } v _ { i } \sum _ { r = 1 } ^ { n } \left[\xi_{m}^{(r 0)}\left(\xi_{m}^{\prime(r i)}-\frac{U_{r i}}{\sqrt{\pi}}\right)-\xi_{m}^{(r i)}\left(\xi_{m}^{\prime(r 0)}-\frac{U_{r 0}}{\sqrt{\pi}}\right)\right.\right.\right. \\
& \left.\left.\left.+\xi_{m}^{(r j)}\left(\xi_{m}^{\prime(r k)}-\frac{U_{r k}}{\sqrt{\pi}}\right)-\xi_{m}^{(r k)}\left(\xi_{m}^{\prime}(r j)-\frac{U_{r j}}{\sqrt{\pi}}\right)\right]\right\}\right) .
\end{aligned}
$$

Setting $\xi_{m}^{\prime \prime}{ }^{(r i)}=\xi_{m}^{\prime(r i)}-\frac{U_{r i}}{\sqrt{\pi}}$, we first integrate with respect to $\xi_{m}^{(r i)}$, which yields

$$
J_{m}=E\left(\exp \left(-\frac{1}{2 m^{2}} \sum_{r=1}^{n} \sum_{i=0}^{3}\left(\mu_{m}^{(r i)}\right)^{2}\right)\right) \text {, }
$$

where

$$
\mu_{m}^{(r)}=\left(\begin{array}{c}
\mu_{m}^{(r 0)} \\
\mu_{m}^{(r 1)} \\
\mu_{m}^{(r 2)} \\
\mu_{m}^{(r 3)}
\end{array}\right)=4\left(\begin{array}{cccc}
0 & v_{1} & v_{2} & v_{3} \\
-v_{1} & 0 & v_{3} & -v_{2} \\
-v_{2} & -v_{3} & 0 & v_{1} \\
-v_{3} & v_{2} & -v_{1} & 0
\end{array}\right)\left(\begin{array}{c}
\xi_{m}^{\prime \prime}(r 0) \\
\xi_{m}^{\prime \prime}(r 1) \\
\xi_{m}^{\prime \prime}(r 2) \\
\xi_{m}^{\prime \prime}(r 3)
\end{array}\right)=a \xi_{m}^{\prime \prime}(r)
$$

written briefly as

$$
\mu_{m}=A \xi_{m}^{\prime \prime}
$$

The skew-symmetric matrix $A$ is more simple than that in [7], which makes the calculation from now on easier than that in [7], and causes the 
heat kernel of the quaternionic Heisenberg groups to be more simple and more concrete than those of the general nilpotent Lie groups of step two.

Thus

$$
\sum_{r=1}^{n} \sum_{i=0}^{3}\left(\mu_{m}^{(r i)}\right)^{2}=\left(A \mu_{m}^{\prime \prime}, A \mu_{m}^{\prime \prime}\right)=-\left(A^{2} \mu_{m}^{\prime \prime}, \mu_{m}^{\prime \prime}\right) .
$$

Let $B=\frac{1}{m^{2}} A^{2}$, as in [7]. Then we have

$$
\begin{aligned}
J_{m} & =\frac{1}{(2 \pi)^{2 n}} \int_{\mathbf{R}^{4 n}} \exp \frac{1}{2}\left[\left(B \xi_{m}^{\prime \prime}, \xi_{m}^{\prime \prime}\right)-\left(\xi_{m}^{\prime \prime}-\frac{U_{m}}{\sqrt{\pi}}, \xi_{m}^{\prime \prime}-\frac{U_{m}}{\sqrt{\pi}}\right)\right] d \xi_{m}^{\prime \prime} \\
& =\exp \left(-\frac{\left|U_{m}\right|^{2}}{2 \pi}+\frac{1}{2 \pi}{ }^{t} U_{m}\left(I_{4 n}-B\right)^{-1} U_{m}\right) / \sqrt{\operatorname{det}\left(I_{4 n}-B\right)} .
\end{aligned}
$$

This yields

$$
E_{0}\left(\exp \left(\sqrt{-1} \sum_{j=1}^{3} v_{j} \sum_{r=1}^{n} \sum_{i=0}^{3} x_{r i}(s)\right) \mid x_{r i}(s)=x_{r i}\right)=\prod_{m=1}^{\infty} \psi_{s}\left(\frac{1}{m} A, \mathbf{x}\right) .
$$

Thus the proof of Theorem 2.2 is finished.

We may explicitly give the diagonalization by $2 \times 2$ block matrices of the skew-symmetric matrix $A$ as follows: Let $\rho=\sqrt{v_{1}^{2}+v_{2}^{2}+v_{3}^{2}}, \sigma=\sqrt{v_{2}^{2}+v_{3}^{2}}$, and set

$$
Y_{0}=\left(\begin{array}{l}
1 \\
0 \\
0 \\
0
\end{array}\right), Y_{1}=\frac{1}{\rho}\left(\begin{array}{c}
0 \\
v_{1} \\
v_{2} \\
v_{3}
\end{array}\right), Y_{2}=\frac{1}{\rho \sigma}\left(\begin{array}{c}
0 \\
-\sigma^{2} \\
v_{1} v_{2} \\
v_{1} v_{3}
\end{array}\right), Y_{3}=\frac{1}{\sigma}\left(\begin{array}{c}
0 \\
0 \\
-v_{3} \\
v_{2}
\end{array}\right) .
$$

It is easy to see that these vectors are orthonormal and

$$
a Y_{0}=-4 \rho Y_{1}, a Y_{1}=4 \rho Y_{0}, a Y_{2}=-4 \rho Y_{3}, a Y_{3}=4 \rho Y_{2} .
$$

Now we introduce an orthogonal matrix $\omega=\left(Y_{0}, Y_{1}, Y_{2}, Y_{3}\right)$. It is readily seen that

$$
{ }^{t} \omega a \omega=\operatorname{diag}\left(\left(\begin{array}{cc}
0 & 4 \rho \\
-4 \rho & 0
\end{array}\right),\left(\begin{array}{cc}
0 & 4 \rho \\
-4 \rho & 0
\end{array}\right)\right)=p .
$$

Furthermore, if we set

$$
\Omega=\operatorname{diag}(\underbrace{\omega, \ldots, \omega}_{n})
$$

then

$$
{ }^{t} \Omega A \Omega=\operatorname{diag}(\underbrace{\left(\begin{array}{cc}
0 & 4 \rho \\
-4 \rho & 0
\end{array}\right), \ldots,\left(\begin{array}{cc}
0 & 4 \rho \\
-4 \rho & 0
\end{array}\right)}_{2 n})=P .
$$

Now we can give the explicit expression of the heat kernel: 
Theorem 2.3. The heat kernel on the quaternionic Heisenberg group $\mathbf{H} H^{n}$ is given by

$$
\begin{aligned}
p_{s}(\mathbf{x}, \mathbf{u})= & p_{s}(e,(\mathbf{x}, \mathbf{u})) \\
= & (2 \pi s)^{2 n}(2 \pi)^{-(4 n+3)} \int_{\mathbf{R}^{3}} \exp \left(-\left(\sqrt{-1} \mathbf{u} \cdot \mathbf{v}+|\mathbf{x}|^{2} \rho \operatorname{coth} 2 s \rho\right)\right) \\
& \cdot\left(\frac{2 s \rho}{\sinh 2 s \rho}\right)^{2 n} d \mathbf{v} .
\end{aligned}
$$

Proof. After the orthogonal transformation represented by matrix $\Omega$, Theorem 2.2 can be rewritten as

(1)

$$
\begin{aligned}
& \hat{p}_{s}(e, .)(\mathbf{y}, \mathbf{v}) \\
& =(2 \pi s)^{-2 n} \int_{\mathbf{R}^{4 n}} \exp \left(\sqrt{-1} \sum_{r=1}^{n} \sum_{i=0}^{3} y_{r i} x_{r i}-\frac{|\mathbf{x}|^{2}}{2 s}\right) \\
& \cdot \prod_{m=1}^{\infty} \exp \left[\frac{1}{s}\left(-|\mathbf{x}|^{2}+{ }^{t} \mathbf{x} \Omega\left(I_{4 n}-\frac{s^{2} P^{2}}{4 \pi^{2} m^{2}}\right)^{-1}{ }^{t} \Omega \mathbf{x}\right)\right] \\
& \quad \cdot \operatorname{det}\left(I_{4 n}-\frac{s P}{2 \pi m}\right)^{-1} d \mathbf{x} .
\end{aligned}
$$

It is easy to verify that

$$
\begin{gathered}
\operatorname{det}\left(I_{4 n}-\frac{s P}{2 \pi m}\right)=\left(1+\frac{4 s^{2} \rho^{2}}{\pi^{2} m^{2}}\right)^{2 n}, \\
{ }^{t} \Omega \mathbf{x}=\left({ }^{t} \omega \mathbf{x}_{1}, \ldots,{ }^{t} \omega \mathbf{x}_{n}\right), \\
\left({ }^{t} \omega \mathbf{x}_{r}\right)_{0}^{2}+\left({ }^{t} \omega \mathbf{x}_{r}\right)_{1}^{2}=\frac{1}{\rho^{2}}\left[\rho^{2} x_{r 0}^{2}+\left(\sum_{i=1}^{3} v_{i} x_{r i}\right)^{2}\right], \\
\left({ }^{t} \omega \mathbf{x}_{r}\right)_{2}^{2}+\left({ }^{t} \omega \mathbf{x}_{r}\right)_{3}^{2} \\
=\frac{1}{\rho^{2}}\left[\left(-\sigma x_{r 1}+\frac{v_{1} v_{2}}{\sigma} x_{r 2}+\frac{v_{1} v_{3}}{\sigma} x_{r 3}\right)^{2}+\left(\frac{-\rho v_{3}}{\sigma} x_{r 2}+\frac{\rho v_{2}}{\sigma} x_{r 3}\right)^{2}\right] \\
=\frac{1}{\rho^{2}}\left[\rho^{2} \sum_{i=1}^{3} x_{r i}^{2}-\left(\sum_{i=1}^{3} v_{i} x_{r i}\right)^{2}\right],
\end{gathered}
$$

and hence

$$
\sum_{i=0}^{3}\left({ }^{t} \omega \mathbf{x}_{r}\right)_{i}^{2}=\sum_{i=0}^{3} x_{r i}^{2},\left|{ }^{t} \Omega \mathbf{x}\right|^{2}=|\mathbf{x}|^{2} .
$$


From the expression of matrix $P$ we have

$$
\begin{aligned}
& \exp \left[\frac{1}{s}\left(-|\mathbf{x}|^{2}+{ }^{t} \mathbf{x} \Omega\left(I_{4 n}-\frac{s^{2} P^{2}}{4 \pi^{2} m^{2}}\right)^{-1}{ }^{t} \Omega \mathbf{x}\right)\right] \\
& =\exp \left\{\frac{1}{s} \sum_{r=1}^{n}\left(-\left|\mathbf{x}_{r}\right|^{2}+{ }^{t} \mathbf{x}_{r} \omega\left(I_{4}-\frac{s^{2} p^{2}}{4 \pi^{2} m^{2}}\right)^{-1}{ }^{t} \omega \mathbf{x}_{r}\right)\right\} \\
& =\exp \left\{\frac{1}{s} \sum_{r=1}^{n} \sum_{i=0}^{3}\left(-1+\frac{\pi^{2} m^{2}}{\pi^{2} m^{2}+4 s^{2} \rho^{2}}\right) x_{r i}^{2}\right\},
\end{aligned}
$$

since

$$
\frac{u}{\sinh u}=\prod_{m=1}^{\infty}\left(1+\frac{u^{2}}{\pi^{2} m^{2}}\right)^{-1},
$$

and

$$
\operatorname{coth} u=\frac{1}{u}+\sum_{m=1}^{\infty} \frac{2 u}{\pi^{2} m^{2}+u^{2}},
$$

therefore

$$
\begin{aligned}
& \prod_{m=1}^{\infty} \exp \left[\frac{1}{s}\left(-|\mathbf{x}|^{2}+{ }^{t} \mathbf{x} \Omega\left(I-\frac{s^{2} P^{2}}{4 \pi^{2} m^{2}}\right)^{-1} \Omega \mathbf{x}\right)\right] \operatorname{det}\left(I-\frac{s P}{2 \pi m}\right)^{-1} \\
& =\exp \left(\frac{1}{s} \sum_{r=1}^{n} \sum_{i=0}^{3} \sum_{m=1}^{\infty} \frac{-4 s^{2} \rho^{2}}{\pi^{2} m^{2}+4 s^{2} \rho^{2}} x_{r i}^{2}\right) \prod_{m=1}^{\infty}\left(1+\frac{4 s^{2} \rho^{2}}{\pi^{2} m^{2}}\right)^{-2 n} \\
& =\exp \left(\frac{|\mathbf{x}|^{2}}{2 s}(1-2 s \rho \cosh 2 s \rho)\right)\left(\frac{2 s \rho}{\sinh 2 s \rho}\right)^{2 n} .
\end{aligned}
$$

Substituting this equality into the right-hand side of (1) and taking the Euclidean Fourier transform, we obtain at once the desired result.

\section{The Green functions of the quaternionic Heisenberg groups.}

It is known that the Green function can be derived from the heat kernel by the formula

$$
G(\mathbf{g})=\int_{0}^{+\infty} p_{s}(e, \mathbf{g}) d s .
$$


So using Theorem 2.3, the Green function of the quaternionic Heisenberg group can be written as

$$
\begin{aligned}
G(\mathbf{x}, \mathbf{u})= & \int_{0}^{+\infty} p_{s}(e,(\mathbf{x}, \mathbf{u})) d s \\
= & (2 \pi)^{-(6 n+3)} \int_{0}^{+\infty} s^{-2 n} d s \int_{\mathbf{R}^{3}} \exp \left(-\left(\sqrt{-1} \mathbf{v} \cdot \mathbf{u}+|\mathbf{x}|^{2} \rho \operatorname{coth} 2 s \rho\right)\right) \\
& \cdot\left(\frac{2 s \rho}{\sinh 2 s \rho}\right)^{2 n} \prod_{j=1}^{3} d v_{j} .
\end{aligned}
$$

First by performing the change of variables $v_{j} \rightarrow s v_{j}$, then it follows that $\rho \rightarrow s \rho$, and

$$
\begin{aligned}
G(\mathbf{x}, \mathbf{u}) & (2 \pi)^{-(6 n+3)} \int_{0}^{+\infty} s^{-(2 n+3)} d s \int_{\mathbf{R}^{3}} \exp \left\{\frac{1}{s}\left(\sqrt{-1} \mathbf{v} \cdot \mathbf{u}+|\mathbf{x}|^{2} \rho \operatorname{coth} 2 \rho\right)\right\} \\
& \cdot\left(\frac{2 \rho}{\sinh 2 \rho}\right)^{2 n} \prod_{j=1}^{3} d v_{j} \\
= & (2 \pi)^{-(6 n+3)} \Gamma(2 n+2) \int_{\mathbf{R}^{3}}\left(|\mathbf{x}|^{2} \operatorname{coth} 2 \rho+\sqrt{-1} \mathbf{v} \cdot \mathbf{u}\right)^{-(2 n+2)} \\
& \cdot\left(\frac{2 \rho}{\sinh 2 \rho}\right)^{2 n} \prod_{j=1}^{3} d v_{j},
\end{aligned}
$$

where $\Gamma($.$) is Euler's Gamma-function.$

In Euclidean space $\mathbf{R}^{3}$, we use polar coordinates and let the positive direction of the $z$-axis coincide with that of vector $\mathbf{u}$, i.e., we set

$$
\mathbf{u}=|\mathbf{u}|(0,0,1), \mathbf{v}=\rho(\sin \theta \cos \varphi, \sin \theta \sin \varphi, \cos \theta)
$$




$$
\begin{aligned}
G(\mathbf{x}, \mathbf{u}) & 2^{n}(2 \pi)^{-(6 n+3)} \Gamma(2 n+2) \int_{0}^{+\infty} \rho^{2}\left(\frac{\rho}{\sinh 2 \rho}\right)^{2 n} d \rho \\
& \cdot \int_{0}^{2 \pi} \int_{0}^{\pi}\left(|\mathbf{x}|^{2} \rho \operatorname{coth} 2 \rho+\sqrt{-1} \rho|\mathbf{u}| \cos \theta\right)^{-(2 n+2)} \sin \theta d \theta d \phi \\
= & 2^{2 n}(2 \pi)^{-(6 n+2)} \frac{\Gamma(2 n+2)}{2 n+1} \int_{0}^{+\infty}\left(\frac{1}{\sinh 2 \rho}\right)^{2 n} \frac{1}{\sqrt{-1}|\mathbf{u}|} \\
& \cdot\left[\left(|\mathbf{x}|^{2} \operatorname{coth} 2 \rho-\sqrt{-1}|\mathbf{u}|\right)^{-(2 n+1)}-\left(|\mathbf{x}|^{2} \operatorname{coth} 2 \rho+\sqrt{-1}|\mathbf{u}|\right)^{-(2 n+1)}\right] d \rho .
\end{aligned}
$$

For $n=1$ it is easy to complete the last integration, and we obtain:

Proposition 3.1. The Green function $G(\mathbf{x}, \mathbf{u})$ of the quaternionic Heisenberg group $\mathbf{H} H^{1}$ is

$$
G(\mathbf{x}, \mathbf{u})=4(2 \pi)^{-8}\left(|\mathbf{x}|^{4}+|\mathbf{u}|^{2}\right)^{-2} .
$$

Proof. When $n=1$, performing the integral in the right-hand side of Equation (2) gives us

$$
\begin{aligned}
G(\mathbf{x}, \mathbf{u})= & (2 \pi)^{-8} \frac{1}{\sqrt{-1}|\mathbf{u}||\mathbf{x}|^{2}} \\
& \cdot\left[\left(|\mathbf{x}|^{2} \operatorname{coth} 2 \rho-\sqrt{-1}|\mathbf{u}|\right)^{-2}-\left(|\mathbf{x}|^{2} \operatorname{coth} 2 \rho+\sqrt{-1}|\mathbf{u}|\right)^{-2}\right]_{\rho=0}^{\rho=+\infty} \\
= & 4(2 \pi)^{-8}\left(|\mathbf{x}|^{4}+|\mathbf{u}|^{2}\right)^{-2} .
\end{aligned}
$$

For general $n \in \mathbf{N}$, there is some difficulty to evaluate the integration in Equation (2), while the above proposition and the corresponding results for the Heisenberg groups [5] motivate us to pose:

Theorem 3.2. The Green functions $G(\mathbf{x}, \mathbf{u})$ of the quaternionic Heisenberg groups $\mathbf{H} H^{n}$ are

$$
G(\mathbf{x}, \mathbf{u})=c_{n}\left(|\mathbf{x}|^{4}+|\mathbf{u}|^{2}\right)^{-(n+1)}
$$

where

$$
c_{n}^{-1}=4(n+1)(n+2) \int_{\mathbf{H} H^{n}}|\mathbf{x}|^{2}\left(|\mathbf{x}|^{4}+|\mathbf{u}|^{2}+1\right)^{-(n+3)} d(\mathbf{x}, \mathbf{u}) .
$$

The method of proof is completely analogous to that for the Heisenberg groups [5]. 


\section{Riesz transforms on the quaternionic Heisenberg groups.}

In this section we shall study the uniformly boundedness of the Riesz transforms with respect to the dimensions of the quaternionic Heisenberg groups.

Stein [11] first studied the Riesz transforms on Euclidean spaces. Afterward various authors investigated the Riesz transform on Riemannian manifolds. Although the boundedness of the Riesz transform on every nilpotent Lie group is well-known [10]. It was Coulhon [3] et al. who first showed the uniform boundedness of Riesz transforms with respect to the dimensions of the Heisenberg groups.

In our investigation there are many properties analogous to that in [3], and for completeness we quote briefly these points. One part which differs, however, is the so called "main estimate" in [3], so this part is presented in detail.

4.1. The vector of the Riesz transforms. For the quaternionic Heisenberg groups, the skew-adjoint Riesz transforms are defined analogously to that in $[\mathbf{3}]$ by

$$
\widetilde{R}_{r i}=X_{r i} \triangle^{-1 / 2}+\triangle^{-1 / 2} X_{r i}, 1 \leq r \leq n, 0 \leq i \leq 3 .
$$

In [3] Coulhon et al. proved the following results:

Lemma 4.1. The skew-adjoint Riesz transform has the expression

$$
\widetilde{R}_{r i} f(\mathbf{x}, \mathbf{u})=\int_{\mathbf{H} H^{n}} X_{r i} p_{1}(e,(\mathbf{y}, \mathbf{v})) \mathcal{H}_{(\mathbf{y}, \mathbf{v})} f(\mathbf{x}, \mathbf{u}) d(\mathbf{y}, \mathbf{v}),
$$

where

$$
\mathcal{H}_{(\mathbf{y}, \mathbf{v})} f(\mathbf{x}, \mathbf{u})=\int_{0}^{+\infty}\left[f\left((\mathbf{x}, \mathbf{u}) \delta_{t}\left((\mathbf{y}, \mathbf{v})^{-1}\right)\right)-f\left((\mathbf{x}, \mathbf{u}) \delta_{t}(\mathbf{y}, \mathbf{v})\right)\right] \frac{d t}{t} .
$$

In fact, this formula is valid for every stratified Lie group.

Lemma 4.2. For every $p \in(1,+\infty)$, there exists $c>0$ depending only on $p$, such that

$$
\left\|\mathcal{H}_{(\mathbf{y}, \mathbf{v})}\right\|_{p \rightarrow p \leq c}, \forall n \in \mathbf{N}, \forall(\mathbf{y}, \mathbf{v}) \in \mathbf{H} H^{n} .
$$

Lemma 4.3. There exists $c^{\prime}>0$ such that

$$
\left\|X_{r i} p_{1}\right\|_{L^{1}\left(\mathbf{H} H^{n}\right)} \leq c^{\prime}, \forall n \in \mathbf{N}, \forall 1 \leq r \leq n, 0 \leq i \leq 3 .
$$

This follows from the fact that one can express the heat kernels for the quaternionic Heisenberg groups $\mathbf{H} H^{n}$ as $p_{s}^{n}$, then it is easy to verify that

$$
p_{1}^{n}\left(\mathbf{x}_{1}, \ldots, \mathbf{x}_{n}, \mathbf{u}\right)=\left[p_{1}^{1}\left(\mathbf{x}_{1}, .\right) * \cdots * p_{1}^{1}\left(\mathbf{x}_{n}, .\right)\right](\mathbf{u}) .
$$

For $f \in C_{0}^{\infty}\left(\mathbf{H} H^{n}\right)$ and $(\mathbf{x}, \mathbf{u}) \in \mathbf{H} H^{n}$, we define a vector field

$$
\widetilde{R} f(\mathbf{x}, \mathbf{u})=\left(\widetilde{R}_{10} f(\mathbf{x}, \mathbf{u}), \ldots, \widetilde{R}_{n 3} f(\mathbf{x}, \mathbf{u})\right)
$$


and its Euclidean length

$$
|\widetilde{R} f(\mathbf{x}, \mathbf{u})|=\left(\sum_{r=1}^{n} \sum_{i=0}^{3}\left|\widetilde{R}_{r i} f(\mathbf{x}, \mathbf{u})\right|^{2}\right)^{1 / 2} .
$$

Let $S^{n}$ be the unit sphere in the quaternionic Heisenberg group $\mathbf{H} H^{n}$, i.e.,

$$
\begin{aligned}
S^{n}=\left\{\mathbf{h}=(\mathbf{z}, \mathbf{w}) \in \mathbf{H} H^{n} \mid \mathbf{z}=\left(\mathbf{z}_{1}, \ldots, \mathbf{z}_{n}\right) \in \mathbf{H}^{n}, \mathbf{w} \in \mathbf{R}^{3},\right. \\
\left.\left(\sum_{r=1}^{n} \sum_{i=0}^{3} z_{r i}^{2}\right)^{2}+\sum_{j=1}^{3} w_{j}^{2}=1\right\} .
\end{aligned}
$$

As in [3], we can deduce that

$$
|\widetilde{R} f(\mathbf{x}, \mathbf{u})|=\int_{S^{n}} \int_{0}^{+\infty} \sum_{r=1}^{n} \sum_{i=0}^{3} \lambda_{r i} X_{r i} p_{1}\left(\delta_{t}(\mathbf{h})\right) \mathcal{H}_{(\mathbf{h})} f(\mathbf{x}, \mathbf{u}) t^{4 n+5} d t d \sigma(\mathbf{h}),
$$

where $d \sigma(\mathbf{h})$ is the surface element on $S^{n}$, the $\lambda_{r i}$ are dependent on $f$ and $(\mathbf{x}, \mathbf{u})$, and $\sum_{r=1}^{n} \sum_{i=0}^{3} \lambda_{r i}^{2}=1$ (we have also used the fact that $\left.\mathcal{H}_{\delta_{t}(\mathbf{y}, \mathbf{v})}=\mathcal{H}_{(\mathbf{y}, \mathbf{v})}\right)$. Applying Hölder's inequality with respect to $d \sigma$, we get, for $1 / p+1 / q=1$,

$$
\begin{aligned}
& |\widetilde{R} f(\mathbf{x}, \mathbf{u})| \\
& \leq\left\|\int_{0}^{+\infty} \sum_{r=1}^{n} \sum_{i=0}^{3} \lambda_{r i} X_{r i} p_{1}\left(\delta_{t}(\mathbf{h})\right) t^{4 n+5} d t\right\|_{L^{q}(d \sigma(\mathbf{h}))}\left\|\mathcal{H}_{\mathbf{h}} f(\mathbf{x}, \mathbf{u})\right\|_{L^{p}(d \sigma(\mathbf{h}))}
\end{aligned}
$$

After a rotation on $S^{n} \bigcap \mathbf{H}^{n}$, one can send $\sum_{r=1}^{n} \sum_{i=0}^{3} \lambda_{r i} X_{r i}$ to $X_{10}$, and it is clear that the heat kernel $p_{1}\left(\delta_{t}(\mathbf{h})\right)$ is invariant under this rotation, hence

$$
\begin{aligned}
& \left\|\int_{0}^{+\infty} \sum_{r=1}^{n} \sum_{i=0}^{3} \lambda_{r i} X_{r i} p_{1}\left(\delta_{t}(\mathbf{h})\right) t^{4 n+5} d t\right\|_{L^{q}(d \sigma(\mathbf{h}))} \\
& =\left\|\int_{0}^{+\infty} X_{10} p_{1}\left(\delta_{t}(\mathbf{h})\right) t^{4 n+5} d t\right\|_{L^{q}(d \sigma(\mathbf{h}))} \cdot
\end{aligned}
$$

Via Lemma 4.2, the argument analogous to that in [3] implies that there exists $c(p)>0$ such that

$$
\|\| \mathcal{H}_{\mathbf{h}} f(\mathbf{x}, \mathbf{u})\left\|_{L^{p}(d \sigma(\mathbf{h}))}\right\|_{L^{p} d(\mathbf{x}, \mathbf{u})} \leq c(p)\left[\sigma\left(S^{n}\right)\right]^{1 / p}\|f\|_{L^{p}(d \mathbf{x}, \mathbf{u})} .
$$


4.2. The main estimate. Let

$$
\Phi(\mathbf{h})=\int_{0}^{+\infty} X_{10} p_{1}\left(\delta_{t}(\mathbf{h})\right) t^{4 n+5} d t, \mathbf{h} \in S^{n} .
$$

Theorem 2.3 gives us

$p_{1}(\mathbf{x}, \mathbf{u})=\frac{1}{(2 \pi)^{6 n+3}} \int_{\mathbf{R}^{3}} \exp \left\{-\left(\sqrt{-1} \mathbf{v} \cdot \mathbf{u}+|\mathbf{x}|^{2} \rho \operatorname{coth} 2 \rho\right)\right\}\left(\frac{2 \rho}{\sinh 2 \rho}\right)^{2 n} \prod_{j=1}^{3} d v_{j}$.

Since $X_{10}=\frac{\partial}{\partial x_{10}}-\sum_{i=1}^{3} 2 x_{1 i} \frac{\partial}{\partial u_{i}}$, it follows that

(6) $X_{10} p_{1}(\mathbf{x}, \mathbf{u})=\frac{1}{(2 \pi)^{6 n+3}} \int_{\mathbf{R}^{3}}\left(-2 x_{10} \rho \operatorname{coth} 2 \rho+2 \sqrt{-1} \sum_{j=1}^{3} v_{j} x_{1 j}\right)$

$$
\begin{aligned}
& \cdot \exp \left\{-\left(\sqrt{-1} \mathbf{v} \cdot \mathbf{u}+|\mathbf{x}|^{2} \rho \operatorname{coth} 2 \rho\right)\right\}\left(\frac{2 \rho}{\sinh 2 \rho}\right)^{2 n} \prod_{j=1}^{3} d v_{j} \\
= & \frac{2}{(2 \pi)^{6 n+3}}\left(-F_{1}(\mathbf{x}, \mathbf{u})+\sqrt{-1} F_{2}(\mathbf{x}, \mathbf{u})\right),
\end{aligned}
$$

where

$$
\begin{aligned}
F_{1}(\mathbf{x}, \mathbf{u})= & x_{10} \int_{\mathbf{R}^{3}} \exp \left\{-\left(\sqrt{-1} \mathbf{v} \cdot \mathbf{u}+|\mathbf{x}|^{2} \rho \operatorname{coth} 2 \rho\right)\right\} \\
& \cdot\left(\frac{2 \rho}{\sinh 2 \rho}\right)^{2 n} \rho \operatorname{coth} 2 \rho \prod_{j=1}^{3} d v_{j} \\
F_{2}(\mathbf{x}, \mathbf{u})= & \int_{\mathbf{R}^{3}} \exp \left\{-\left(\sqrt{-1} \mathbf{v} \cdot \mathbf{u}+|\mathbf{x}|^{2} \rho \operatorname{coth} 2 \rho\right)\right\} \\
& \cdot\left(\frac{2 \rho}{\sinh 2 \rho}\right)^{2 n} \sum_{j=1}^{3} v_{j} x_{1 j} \prod_{j=1}^{3} d v_{j} .
\end{aligned}
$$

Thus

$$
\begin{aligned}
\Phi(\mathbf{h}) & =\frac{2}{(2 \pi)^{6 n+3}} \int_{0}^{+\infty}\left[-F_{1}\left(\delta_{t}(\mathbf{h})\right)+\sqrt{-1} F_{2}\left(\delta_{t}(\mathbf{h})\right)\right] t^{4 n+5} d t \\
& =\frac{2}{(2 \pi)^{6 n+3}}\left(-\Phi_{1}+\sqrt{-1} \Phi_{2}\right)(\mathbf{h}) .
\end{aligned}
$$


Let $\mathbf{h}=(\mathbf{z}, \mathbf{w})=\left(z_{10}, \ldots, z_{n 3}, w_{1}, w_{2}, w_{3}\right) \in S^{n}$. So we get

$$
\begin{aligned}
\Phi_{1}(\mathbf{h})= & \int_{0}^{+\infty} F_{1}\left(\delta_{t}(\mathbf{h})\right) t^{4 n+5} d t \\
= & z_{10} \int_{\mathbf{R}^{3}} \int_{0}^{+\infty} \exp \left\{-t^{2}\left(\sqrt{-1} \mathbf{v} \cdot \mathbf{w}+|\mathbf{z}|^{2} \rho \operatorname{coth} 2 \rho\right)\right\} \\
& \cdot\left(\frac{2 \rho}{\sinh 2 \rho}\right)^{2 n} \rho \operatorname{coth} 2 \rho t^{4 n+6} d t \prod_{j=1}^{3} d v_{j} \\
= & \frac{1}{2} \Gamma\left(\frac{n+7}{2}\right) z_{10} \int_{\mathbf{R}^{3}}\left(|\mathbf{z}|^{2} \rho \operatorname{coth} 2 \rho+\sqrt{-1} \mathbf{v} \cdot \mathbf{w}\right)^{-\frac{4 n+7}{2}} \\
& \cdot\left(\frac{2 \rho}{\sinh 2 \rho}\right)^{2 n} \rho \operatorname{coth} 2 \rho \prod_{j=1}^{3} d v_{j} .
\end{aligned}
$$

Taking the polar coordinates in $\mathbf{R}^{3}$ as in the proof of Proposition 3.1, the above function becomes

$$
\begin{aligned}
\Phi_{1}(\mathbf{h})= & \frac{1}{2} \Gamma\left(\frac{4 n+7}{2}\right) z_{10} \int_{0}^{+\infty} \int_{0}^{\pi} \int_{0}^{2 \pi}\left(|\mathbf{z}|^{2} \operatorname{coth} 2 \rho+\sqrt{-1}|\mathbf{w}| \cos \theta\right)^{-\frac{4 n+7}{2}} \\
& \cdot\left(\frac{2 \rho}{\sinh 2 \rho}\right)^{2 n} \rho^{-\frac{4 n+1}{2}} \operatorname{coth} 2 \rho \sin \theta d \theta d \phi d \rho \\
= & \frac{2^{2 n}}{4 n+5} 2 \pi \Gamma\left(\frac{4 n+7}{2}\right) z_{10} \int_{0}^{+\infty} \rho \cosh 2 \rho\left(\frac{\rho}{\sinh 2 \rho}\right)^{-3 / 2}\left(\frac{1}{\sqrt{-1}|\mathbf{w}|}\right) \\
& \cdot\left[\left(|\mathbf{z}|^{2} \cosh 2 \rho-\sqrt{-1}|\mathbf{w}| \sinh 2 \rho\right)^{-\frac{4 n+5}{2}}\right. \\
& \left.-\left(|\mathbf{z}|^{2} \cosh 2 \rho+\sqrt{-1}|\mathbf{w}| \sinh 2 \rho\right)^{-\frac{4 n+5}{2}}\right] d \rho .
\end{aligned}
$$

On $S^{n}$ we perform the change of variables given by $|\mathbf{z}|^{2}=\cos \psi,|\mathbf{w}|=$ $\sin \psi$. Then

$$
\begin{aligned}
& \left(|\mathbf{z}|^{2} \cosh 2 \rho-\sqrt{-1}|\mathbf{w}| \sinh 2 \rho\right)^{-\frac{4 n+5}{2}}-\left(|\mathbf{z}|^{2} \cosh 2 \rho+\sqrt{-1}|\mathbf{w}| \sinh 2 \rho\right)^{-\frac{4 n+5}{2}} \\
& =\cosh ^{-\frac{4 n+5}{2}}(2 \rho-\sqrt{-1} \psi)-\cosh ^{-\frac{4 n+5}{2}}(2 \rho+\sqrt{-1} \psi) \\
& =2(4 n+5) \sqrt{-1} \cosh ^{-\frac{4 n+7}{2}} 2 \rho \sinh 2 \rho \psi+O(|\psi|) .
\end{aligned}
$$

Noting that $\frac{|\psi|}{|\mathbf{w}|}=\frac{|\psi|}{|\sin \psi|} \leq c$, we obtain the inequality:

$$
\begin{aligned}
\left|\Phi_{1}(\mathbf{h})\right| \leq & 2^{2 n+1}(2 \pi) \Gamma\left(\frac{4 n+7}{2}\right)\left|z_{10}\right| \\
& \cdot \int_{0}^{+\infty} \cosh ^{-\frac{4 n+5}{2}} 2 \rho \sinh ^{2} 2 \rho\left(\frac{\rho}{\sinh 2 \rho}\right)^{-1 / 2} d \rho .
\end{aligned}
$$


Since $\frac{\sinh 2 \rho}{\rho}=2 \cosh (2 \theta \rho) \leq 2 \cosh 2 \rho$, with $0<\theta<1$, we finally obtain an estimation for $\Phi_{1}(\mathbf{h})$ :

$$
\begin{aligned}
\left|\Phi_{1}(\mathbf{h})\right| & \leq 2^{2 n+3 / 2}(2 \pi) \Gamma\left(\frac{4 n+7}{2}\right)\left|z_{10}\right| \int_{0}^{+\infty} \cosh ^{-2 n} 2 \rho d \rho \\
& \leq c .2^{4 n}(2 \pi) \Gamma\left(\frac{4 n+7}{2}\right)\left|z_{10}\right| B(n, n),
\end{aligned}
$$

where $B(n, n)$ is Euler's Beta-function, since $\int_{0}^{+\infty} \cosh ^{-2 n} 2 \rho d \rho=$ $2^{2 n-2} B(n, n)$.

Now we begin to estimate $\Phi_{2}(\mathbf{h})$. This is different from the case of the Heisenberg groups. In the present situation the method to evaluate $\Phi_{2}(\mathbf{h})$ is not analogous to that for $\Phi_{1}(\mathbf{h})$, so we record its details. Let $\mathbf{z}_{1}^{\prime}=\left(z_{11}, z_{12}, z_{13}\right)$. Then

$$
\begin{aligned}
\Phi_{2}(\mathbf{h})= & \int_{0}^{+\infty} F_{2}\left(\delta_{t}(\mathbf{h})\right) t^{4 n+5} d t \\
= & -\sqrt{-1} \int_{0}^{+\infty} \int_{\mathbf{R}^{3}} \exp \left\{-t^{2}\left(\sqrt{-1} \mathbf{v} \cdot \mathbf{w}+|\mathbf{z}|^{2} \rho \cosh \rho\right)\right\} \\
& \cdot\left(\frac{2 \rho}{\sinh 2 \rho}\right)^{2 n} t^{4 n+6} \sum_{j=1}^{3} v_{j} z_{1 j} d t \prod_{j=1}^{3} d v_{j} \\
= & -\frac{\sqrt{-1}}{2} \Gamma\left(\frac{4 n+7}{2}\right) \int_{\mathbf{R}^{3}}\left(|\mathbf{z}|^{2} \rho \operatorname{coth} 2 \rho+\sqrt{-1} \mathbf{v} \cdot \mathbf{w}\right)^{-\frac{4 n+7}{2}} \\
& \cdot\left(\frac{2 \rho}{\sinh 2 \rho}\right)^{2 n} \mathbf{z}_{1}^{\prime} \cdot \mathbf{v} \prod_{j=1}^{3} d v_{j} .
\end{aligned}
$$

In the w-space we take polar coordinates, and let the positive direction of the $z$-axis coincide with that of the vector $\mathbf{w}$, i.e.,

$$
\begin{aligned}
\mathbf{w} & =|\mathbf{w}|(0,0,1), \\
\mathbf{v} & =\rho(\sin \theta \cos \phi, \sin \theta \sin \phi, \cos \theta), \\
\mathbf{z}_{1}^{\prime} & =\left|\mathbf{z}_{1}^{\prime}\right|\left(\sin \theta^{\prime} \cos \phi^{\prime}, \sin \theta^{\prime} \sin \phi^{\prime}, \cos \theta^{\prime}\right),
\end{aligned}
$$

where $\theta^{\prime}$ is the angle between vectors $\mathbf{w}$ and $\mathbf{z}_{1}^{\prime}$. Therefore 


$$
\begin{aligned}
\Phi_{2}(\mathbf{w})= & -\frac{\sqrt{-1}}{2} \Gamma\left(\frac{4 n+7}{2}\right)\left|\mathbf{z}_{1}^{\prime}\right| \\
& \cdot \int_{0}^{+\infty} \int_{0}^{\pi} \int_{0}^{2 \pi}\left(|\mathbf{z}|^{2} \rho \operatorname{coth} 2 \rho+\sqrt{-1} \rho|\mathbf{w}| \cos \theta\right)^{-\frac{4 n+7}{2}} \\
& \cdot\left(\sin \theta \sin \theta^{\prime} \cos \phi \cos \phi^{\prime}+\sin \theta \sin \theta^{\prime} \sin \phi \sin \phi^{\prime}+\cos \theta \cos \theta^{\prime}\right) \rho^{3} \sin \theta \\
& \cdot\left(\frac{2 \rho}{\sinh 2 \rho}\right)^{2 n} d \phi d \theta d \rho .
\end{aligned}
$$

First, integrating with respect to $\phi$ yields

$$
\begin{aligned}
\Phi_{2}(\mathbf{h})= & -2^{2 n-1}(2 \pi) \sqrt{-1} \Gamma\left(\frac{4 n+7}{2}\right)\left|\mathbf{z}_{1}^{\prime}\right| \cos \theta^{\prime} \\
& \cdot \int_{0}^{+\infty} \int_{0}^{\pi}\left(|\mathbf{z}|^{2} \cosh 2 \rho+\sqrt{-1}|\mathbf{w}| \sinh 2 \rho \cos \theta\right)^{-\frac{4 n+7}{2}} \\
& \cdot\left(\frac{\rho}{\sinh 2 \rho}\right)^{-7 / 2} \rho^{3} \sin \theta \cos \theta d \theta d \rho .
\end{aligned}
$$

Let

$$
K(\rho)=\int_{0}^{\pi}\left(|\mathbf{z}|^{2} \cosh 2 \rho+\sqrt{-1}|\mathbf{w}| \sinh 2 \rho \cos \theta\right)^{-\frac{4 n+7}{2}} \sin \theta \cos \theta d \theta .
$$

Then a simple calculation gives us

$$
\begin{aligned}
K(\rho)= & \frac{1}{\sqrt{-1}|\mathbf{w}| \sinh 2 \rho} \int_{0}^{\pi}\left[\left(|\mathbf{z}|^{2} \cosh 2 \rho+\sqrt{-1}|\mathbf{w}| \sinh 2 \rho \cos \theta\right)^{-\frac{4 n+5}{2}}\right. \\
& \left.-|\mathbf{z}|^{2} \cosh 2 \rho\left(|\mathbf{z}|^{2} \cosh 2 \rho+\sqrt{-1}|\mathbf{w}| \sinh 2 \rho \cos \theta\right)^{-\frac{4 n+7}{2}}\right] \sin \theta d \theta \\
= & \frac{1}{(\sqrt{-1}|\mathbf{w}| \sinh 2 \rho)^{2}}\left\{\frac { 2 } { 4 n + 3 } \left[\left(|\mathbf{z}|^{2} \cosh 2 \rho-\sqrt{-1}|\mathbf{w}| \sinh 2 \rho\right)^{-\frac{4 n+3}{2}}\right.\right. \\
& \left.-\left(|\mathbf{z}|^{2} \cosh 2 \rho+\sqrt{-1}|\mathbf{w}| \sinh 2 \rho\right)^{-\frac{4 n+3}{2}}\right] \\
& -\frac{2}{4 n+5}|\mathbf{z}|^{2} \cosh 2 \rho\left[\left(|\mathbf{z}|^{2} \cosh 2 \rho-\sqrt{-1}|\mathbf{w}| \sinh 2 \rho\right)^{-\frac{4 n+5}{2}}\right. \\
& \left.\left.-\left(|\mathbf{z}|^{2} \cosh 2 \rho+\sqrt{-1}|\mathbf{w}| \sinh 2 \rho\right)^{-\frac{4 n+5}{2}}\right]\right\}
\end{aligned}
$$


Similar to (8), we have

$$
\begin{aligned}
K(\rho) & =\frac{1}{\sqrt{-1}|\mathbf{w}|^{2} \sinh ^{2} 2 \rho}\left\{\frac{2}{4 n+3}\right. \\
& \cdot\left[\cosh ^{-\frac{4 n+3}{2}}(2 \rho-\sqrt{-1} \psi)-\cosh ^{-\frac{4 n+3}{2}}(2 \rho+\sqrt{-1} \psi)\right] \\
& -\frac{2}{4 n+5}|\mathbf{z}|^{2} \cosh 2 \rho \\
& \left.\cdot\left[\cosh ^{-\frac{4 n+5}{2}}(2 \rho-\sqrt{-1} \psi)-\cosh ^{-\frac{4 n+5}{2}}(2 \rho+\sqrt{-1} \psi)\right]+O(\psi)\right\} \\
= & -\frac{4 \sqrt{-1}}{|\mathbf{w}|^{2} \sinh ^{2} 2 \rho}\left\{\cosh ^{-\frac{4 n+5}{2}} 2 \rho \sinh 2 \rho \psi\left(1-|\mathbf{z}|^{\mathbf{2}}\right)+\mathbf{O}(\psi)\right\},
\end{aligned}
$$

and hence we obtain the estimation

$$
\begin{aligned}
|K(\rho)| \leq & c \cdot \frac{1}{|\mathbf{w}| \sinh ^{2} 2 \rho} \mid \cosh ^{-\frac{4 n+5}{2}} 2 \rho \sinh 2 \rho\left(\frac{\sqrt{-1} \psi}{\sin \psi}\right) \\
& -|\mathbf{z}|^{2} \cosh ^{-\frac{4 n+5}{2}} 2 \rho \sinh 2 \rho\left(\frac{\sqrt{-1} \psi}{\sin \psi}\right) \mid \\
\leq & c \frac{\left(1-|\mathbf{z}|^{2}\right)}{|\mathbf{w}| \sinh 2 \rho} \cosh ^{-\frac{4 n+5}{2}} 2 \rho \leq \frac{c}{\sinh 2 \rho} \cosh ^{-\frac{4 n+5}{2}} 2 \rho .
\end{aligned}
$$

The last inequality follows from the fact that $1-|\mathbf{z}|^{2}=\frac{|\mathbf{w}|^{2}}{1+|\mathbf{z}|^{2}} \leq|\mathbf{w}|^{2} \leq 1$, since $(\mathbf{z}, \mathbf{w}) \in S^{n}$. Substituting the estimation of $K(\rho)$ into the expression of $\Phi_{2}(\mathbf{h})$, we finally obtain

$$
\begin{aligned}
\left|\Phi_{2}(\mathbf{h})\right| & \leq c .2^{2 n-1}(2 \pi) \Gamma\left(\frac{4 n+7}{2}\right)\left|\mathbf{z}_{1}^{\prime}\right| \int_{0}^{+\infty} \cosh ^{-\frac{4 n+5}{2}} 2 \rho\left(\frac{\rho}{\sinh 2 \rho}\right)^{-5 / 2} d \rho \\
& \leq c .2^{2 n}(2 \pi) \Gamma\left(\frac{4 n+7}{2}\right)\left|\mathbf{z}_{1}^{\prime}\right| \int_{0}^{+\infty} \cosh ^{-2 n} 2 \rho d \rho \\
& \leq c .2^{4 n}(2 \pi) \Gamma\left(\frac{4 n+7}{2}\right)\left|\mathbf{z}_{1}^{\prime}\right| B(n, n) .
\end{aligned}
$$

Lemma 4.4. The surface measure of the unit sphere $S^{n}$ of the quaternionic Heisenberg group $\mathbf{H} H^{n}$ is

$$
\sigma\left(S^{n}\right)=2 \pi^{2 n+3 / 2} \frac{\Gamma(n)}{\Gamma(2 n) \Gamma(n+3 / 2)} .
$$


Proof. Let $f \in L^{1}\left(\mathbf{H} H^{n}\right)$ and

$$
\begin{aligned}
I(f) & =\int_{\mathbf{H} H^{n}} f(\mathbf{x}, \mathbf{u}) d(\mathbf{x}, \mathbf{u}) \\
& =\int_{0}^{+\infty} \int_{0}^{+\infty} \int_{\Sigma^{4 n-1}} \int_{\Sigma^{2}} f(R \mathbf{z}, \rho \mathbf{w}) R^{4 n-1} \rho^{2} d \mathbf{w} d \mathbf{z} d \rho d R,
\end{aligned}
$$

where

$$
\Sigma^{n-1}=\left\{\mathbf{x}=\left(x_{1}, \ldots, x_{n}\right) \in \mathbf{R}^{n} \mid \sum_{i=1}^{n} x_{i}^{2}=1\right\}
$$

is the unit sphere in Euclidean space $\mathbf{R}^{n}$. Performing the change of variables $R^{2}=l^{2} \cos \theta, \rho=l^{2} \sin \theta$, we get

$$
\begin{aligned}
I(f)= & \int_{0}^{+\infty} \int_{0}^{\pi / 2} \int_{\Sigma^{4 n-1}} \int_{\Sigma^{2}} f\left(l \mathbf{z} \cos ^{1 / 2} \theta, l^{2} \mathbf{w} \sin \theta\right) \\
& \cdot l^{4 n+5} \cos ^{2 n-1} \theta \sin ^{2} \theta d \mathbf{w} d \mathbf{z} d \theta d l .
\end{aligned}
$$

Hence

(11)

$$
\int_{S^{n}} f(\mathbf{h}) d \sigma(\mathbf{h})=\int_{0}^{\pi / 2} \int_{\Sigma^{4 n-1}} \int_{\Sigma^{2}} f\left(\mathbf{z} \cos ^{1 / 2} \theta, \mathbf{w} \sin \theta\right) \cos ^{2 n-1} \theta \sin ^{2} \theta d \mathbf{w} d \mathbf{z} d \theta .
$$

In particular,

$$
\begin{aligned}
\sigma\left(S^{n}\right) & =\int_{0}^{\pi / 2} \int_{\Sigma^{4 n-1}} \int_{\Sigma^{2}} \cos ^{2 n-1} \theta \sin ^{2} \theta d \mathbf{w} d \mathbf{z} d \theta \\
& =\left|\Sigma^{4 n-1}\right| .\left|\Sigma^{2}\right| \int_{0}^{\pi / 2} \cos ^{2 n-1} \theta \sin ^{2} \theta d \theta=\frac{1}{2}\left|\Sigma^{4 n-1}\right| \cdot\left|\Sigma^{2}\right| B(n, 3 / 2) .
\end{aligned}
$$

Then Lemma 4.4 follows from the expression of the surface measure $\left|\Sigma^{n-1}\right|=\frac{2 \pi^{n / 2}}{\Gamma(n / 2)}$ of the unit sphere in Euclidean space $\mathbf{R}^{n}$.

Now we turn to evaluate the following integral:

Lemma 4.5. We have

$$
\int_{S^{n}}\left|z_{10}\right|^{q} d \sigma(\mathbf{h})=\frac{2 \pi^{2 n+1} \Gamma(n+q / 4) \Gamma\left(\frac{q+1}{2}\right)}{\Gamma(n+q / 4+3 / 2) \Gamma(2 n+q / 2)} .
$$

Proof. Let $\Sigma^{4 n-1}=\left\{\mathbf{z}=\left(\mathbf{z}_{1}, \mathbf{z}^{\prime}\right) \in \mathbf{R}^{4} \times\left.\mathbf{R}^{4 n-4}|| \mathbf{z}_{1}\right|^{2}+\left|\mathbf{z}^{\prime}\right|^{2}=1\right\}$. We introduce new polar coordinates by setting $\mathbf{z}_{1}=\mathbf{a} \cos \phi, \mathbf{z}^{\prime}=\mathbf{b} \sin \phi$ with $\mathbf{a} \in \Sigma^{3}, \mathbf{b} \in \Sigma^{4 n-5}, 0 \leq \phi \leq \pi / 2$. Then formula (11) reads as

$$
\begin{aligned}
& \int_{S^{n}} f(\mathbf{h}) d \sigma(\mathbf{h}) \\
& =\int_{0}^{\pi / 2} \int_{0}^{\pi / 2} \int_{\Sigma^{3}} \int_{\Sigma^{4 n-5}} \int_{\Sigma^{2}} f\left(\mathbf{a} \cos ^{1 / 2} \theta \cos \phi, \mathbf{b} \cos ^{1 / 2} \theta \sin \phi, \mathbf{w} \sin \theta\right) \\
& \quad \cdot \cos ^{2 n-1} \theta \sin ^{2} \theta \cos ^{3} \phi \sin ^{4 n-5} \phi d \mathbf{w} d \mathbf{b} d \mathbf{a} d \theta d \phi .
\end{aligned}
$$


In particular, we have

$$
\begin{aligned}
\int_{S^{n}}\left|z_{10}\right|^{q} d \sigma(\mathbf{h})= & \int_{0}^{\pi / 2} \int_{0}^{\pi / 2} \int_{\Sigma^{3}} \int_{\Sigma^{4 n-5}} \int_{\Sigma^{2}}\left|a_{0} \cos ^{1 / 2} \theta \cos \phi\right|^{q} \\
& \cdot \cos ^{2 n-1} \theta \sin ^{2} \theta \cos ^{3} \phi \sin ^{4 n-5} \phi d \mathbf{w} d \mathbf{b} d \mathbf{a} d \theta d \phi .
\end{aligned}
$$

Furthermore, on the unit sphere $\Sigma^{3}$ of the a-space, we employ the spherical coordinates, i.e.,

$$
\mathbf{a}=\left(\begin{array}{c}
a_{0} \\
a_{1} \\
a_{2} \\
a_{3}
\end{array}\right)=\left(\begin{array}{c}
\sin \psi_{1} \\
\cos \psi_{1} \sin \psi_{2} \\
\cos \psi_{1} \cos \psi_{2} \sin \phi_{3} \\
\cos \psi_{1} \cos \psi_{2} \cos \psi_{3}
\end{array}\right),
$$

with $-\pi / 2 \leq \psi_{1}, \psi_{2} \leq \pi / 2$ and $-\pi \leq \psi_{3} \leq \pi$. Then the integral on $\Sigma^{3}$ in (12) becomes

$$
\begin{aligned}
& \int_{\Sigma^{3}}\left|a_{0}\right|^{q} d \mathbf{a} \\
& =\int_{-\pi / 2}^{\pi / 2} \int_{-\pi / 2}^{\pi / 2} \int_{-\pi}^{\pi}\left|\sin \psi_{1}\right|^{q} \cos ^{2} \psi_{1} \cos \psi_{2} d \psi_{3} d \psi_{2} d \psi_{1}=4 \pi B\left(\frac{q+1}{2}, \frac{3}{2}\right) .
\end{aligned}
$$

Finally we obtain

$$
\begin{aligned}
& \int_{S^{n}}\left|z_{10}\right|^{q} d \sigma(\mathbf{h}) \\
& =4 \pi B\left(\frac{q+1}{2}, \frac{3}{2}\right) \\
& \cdot \int_{0}^{\pi / 2} \int_{0}^{\pi / 2} \int_{\Sigma^{4 n-5}} \int_{\Sigma^{2}} \cos ^{2 n+q / 2-1} \theta \sin ^{2} \theta \cos ^{q+3} \phi \sin ^{4 n-5} \phi d \mathbf{w} d \mathbf{b} d \phi d \theta \\
& =\pi\left|\Sigma^{4 n-5}\right|\left|\Sigma^{2}\right| B\left(\frac{q+1}{2}, \frac{3}{2}\right) B\left(n+\frac{q}{4}, \frac{3}{2}\right) B\left(\frac{q+4}{2}, 2 n-2\right),
\end{aligned}
$$

as required.

Corollary 4.6. We also have

$$
\int_{S^{n}}\left|\mathbf{z}_{1}^{\prime}\right|^{q} d \sigma(\mathbf{h})=\frac{q+1}{2}\left(\frac{\pi^{2 n+1} \Gamma\left(n+\frac{q}{4}\right) \Gamma\left(\frac{q+1}{2}\right)}{\Gamma\left(n+\frac{q+6}{4}\right) \Gamma\left(2 n+\frac{q}{2}\right)}\right) .
$$

Proof. Since

$$
\left|\mathbf{z}_{1}^{\prime}\right|=\left(\sum_{i=1}^{3} a_{1 i}^{2}\right)^{1 / 2} \cos ^{1 / 2} \theta \cos \phi=\cos \psi_{1} \cos ^{1 / 2} \theta \cos \phi
$$


an analogous calculation to that in the proof of Lemma 4.5 gives:

$$
\begin{aligned}
& \int_{S^{n}}\left|\mathbf{z}_{1}^{\prime}\right|^{q} d \sigma(\mathbf{h}) \\
& =\pi\left|\Sigma^{4 n-5}\right|\left|\Sigma^{2}\right| B\left(\frac{q+3}{2}, \frac{1}{2}\right) B\left(n+\frac{q}{4}, \frac{1}{2}\right) B\left(\frac{q+4}{2}, 2 n-2\right),
\end{aligned}
$$

which is exactly the conclusion of Corollary 4.6.

Lemma 4.7. There exists a constant $c=c(q)>0$, such that, $\forall n \in \mathbf{N}$,

$$
\left(\int_{S^{n}}|\Phi(\mathbf{h})|^{q} d \sigma(\mathbf{h})\right)^{1 / q} \leq c\left[\sigma\left(S^{n}\right)\right]^{-1 / p} .
$$

Proof. We know ([10]) that when $x \rightarrow+\infty$,

$$
\frac{\Gamma(x)}{\Gamma(x+a)} \sim x^{-a}, \quad a>0
$$

and Stirling's formula

$$
\Gamma(x) \sim \sqrt{2 \pi} x^{x-1 / 2} \exp (-x) .
$$

From Equations (7), (9), (10), it follows that

$$
|\Phi(\mathbf{h})| \leq c \cdot \frac{2^{4 n}}{(2 \pi)^{6 n+2}} \Gamma(2 n+7 / 2) B(n, n) \max \left\{\left|z_{10}\right|,\left|\mathbf{z}_{1}^{\prime}\right|\right\}
$$

Via Lemmas 4.4 and 4.5 and Corollary 4.6 we get 


$$
\begin{aligned}
& {\left[\sigma\left(S^{n}\right)\right]^{1 / p} \cdot\left(\int_{S^{n}}|\Phi(\mathbf{h})|^{q} d \sigma(\mathbf{h})\right)^{1 / q}} \\
& \leq c \cdot \frac{2^{4 n}}{(2 \pi)^{6 n+2}} \Gamma(2 n+7 / 2) B(n, n) \\
& \quad \cdot\left(\frac{\pi^{2 n+1} \Gamma(n+q / 4) \Gamma\left(\frac{q+1}{2}\right)}{\Gamma\left(n+\frac{q+6}{4}\right) \Gamma(2 n+q / 2)}\right)^{1 / q}\left(\frac{2 \pi^{2 n+3 / 2} \Gamma(n)}{\Gamma(n+3 / 2) \Gamma(2 n)}\right)^{1 / p} \\
& \leq c \cdot \frac{2^{4 n}}{(2 \pi)^{4 n+1} \Gamma(2 n+7 / 2) B(n, n)} \\
& \quad \cdot\left(\frac{\Gamma(n+q / 4)}{\Gamma(n+q / 4+3 / 2) \Gamma(2 n+q / 2)}\right)^{1 / q}\left(\frac{\Gamma(n)}{\Gamma(n+3 / 2) \Gamma(2 n)}\right)^{1 / p} \\
& \leq c . \Gamma(2 n+7 / 2) B(n, n)\left(\frac{1}{\Gamma(2 n+q / 2)}\right)^{1 / q}\left(\frac{1}{\Gamma(2 n)}\right)^{1 / p} \\
& \leq c . B(n, n)\left(\frac{\Gamma(2 n+7 / 2)}{\Gamma(2 n)}\right)\left(\frac{\Gamma(2 n)}{\Gamma(2 n+q / 2)}\right)^{1 / q} \\
& \leq c .(2 n)^{3} B(n, n) \\
& \leq c .(2 n)^{3}\left(\frac{\left(\sqrt{2 \pi} n^{n-1 / 2} \exp (-n)\right)^{2}}{\sqrt{2 \pi}(2 n)^{2 n-1 / 2} \exp (-2 n)}\right)^{1 / 2} \\
& \leq c,
\end{aligned}
$$

which completes the proof of Lemma 4.7. (In the proof of this Lemma, we have used formulas (13) and (14).)

Due to Lemma 4.7 and the inequalities (3), (4), (5) we have proved:

Proposition 4.8. For every $p \in(0,+\infty)$, there exsists a constant $c(p)>0$, such that

$$
\|\widetilde{R} f\|_{L^{p}\left(\mathbf{H} H^{n}\right)} \leq c(p)\|f\|_{L^{p}\left(\mathbf{H} H^{n}\right)}, \forall f \in C_{0}^{\infty}\left(\mathbf{H} H^{n}\right), \forall n \in \mathbf{N} .
$$

4.3. The full Riesz transforms. We consider the full Riesz transform $\mathbf{R} f(\mathbf{x}, \mathbf{u})=\left(R_{10} f(\mathbf{x}, \mathbf{u}), \ldots, R_{n 3} f(\mathbf{x}, \mathbf{u})\right)$, where

$$
\begin{aligned}
R_{r i} f(\mathbf{x}, \mathbf{u}) & =\left(X_{r i} \triangle^{-1 / 2} f\right)(\mathbf{x}, \mathbf{u}) \\
& =\lim _{\varepsilon \rightarrow 0^{+}} \int_{\mathbf{H} H^{n}} X_{r i} p_{1}(\mathbf{y}, \mathbf{v}) \int_{\varepsilon}^{1 / \varepsilon} f\left((\mathbf{x}, \mathbf{u}) \delta_{t}\left((\mathbf{y}, \mathbf{v})^{-1}\right)\right) \frac{d t}{t} d(\mathbf{y}, \mathbf{v}) .
\end{aligned}
$$

Analogous to (6), we have

$$
X_{r i} p_{1}(\mathbf{y}, \mathbf{v})=\frac{2}{(2 \pi)^{6 n+3}}\left(-F_{1}+\sqrt{-1} F_{2}\right)(\mathbf{y}, \mathbf{v})=\frac{2}{(2 \pi)^{6 n+3}} F(\mathbf{y}, \mathbf{v}),
$$


hence

$$
\begin{aligned}
\left(X_{r i} \triangle^{-1 / 2} f\right)(\mathbf{x}, \mathbf{u}) & \\
= & -\frac{2}{(2 \pi)^{6 n+3}} \lim _{\varepsilon \rightarrow 0^{+}} \int_{\mathbf{H} H^{n}}\left(F_{1}-\sqrt{-1} F_{2}\right)(\mathbf{y}, \mathbf{v}) \\
& \cdot \int_{\varepsilon}^{1 / \varepsilon} f\left((\mathbf{x}, \mathbf{u}) \delta_{t}\left((\mathbf{y}, \mathbf{v})^{-1}\right)\right) \frac{d t}{t} d(\mathbf{y}, \mathbf{v}),
\end{aligned}
$$

where

$$
\begin{aligned}
F_{1}(\mathbf{x}, \mathbf{u})= & x_{10} \int_{\mathbf{R}^{3}} \exp \left\{-\left(\sqrt{-1} \mathbf{v} \cdot \mathbf{u}+|\mathbf{x}|^{2} \rho \operatorname{coth} 2 \rho\right)\right\} \\
& \cdot\left(\frac{2 \rho}{\sinh 2 \rho}\right)^{2 n} \rho \operatorname{coth} 2 \rho \prod_{j=1}^{3} d v_{j}, \\
F_{2}(\mathbf{x}, \mathbf{u})= & \int_{\mathbf{R}^{3}} \exp \left\{-\left(\sqrt{-1} \mathbf{v} \cdot \mathbf{u}+|\mathbf{x}|^{2} \rho \operatorname{coth} 2 \rho\right)\right\} \\
& \cdot\left(\frac{2 \rho}{\sinh 2 \rho}\right)^{2 n} \sum_{j=1}^{3} x_{1 j} v_{j} \prod_{j=1}^{3} d v_{j} .
\end{aligned}
$$

From these equations it is easy to see that

$$
F(-\mathbf{x}, \mathbf{u})=-F(\mathbf{x}, \mathbf{u}),
$$

and hence we can write

$$
\begin{aligned}
\left(X_{r i} \triangle^{-1 / 2} f\right)(\mathbf{x}, \mathbf{u}) \\
=\frac{1}{(2 \pi)^{6 n+3}} \int_{\mathbf{H} H^{n}} F(\mathbf{y}, \mathbf{v}) \\
\quad \cdot \int_{0}^{+\infty}\left[f\left((\mathbf{x}, \mathbf{u}) \delta_{t}(-\mathbf{y},-\mathbf{v})\right)-f\left((\mathbf{x}, \mathbf{u}) \delta_{\mathbf{t}}(\mathbf{y},-\mathbf{v})\right)\right] \frac{\mathbf{d t}}{\mathbf{t}} \mathbf{d}(\mathbf{y}, \mathbf{v}) .
\end{aligned}
$$

Similar arguments to those in [3] show that:

Theorem 4.9. For every $p \in(1,+\infty)$, there exists a constant $c=c(p)>0$, such that for all $n \in \mathbf{N}$,

$$
\frac{1}{c}\|f\|_{L^{p}\left(\mathbf{H} H^{n}\right)} \leq\||\mathbf{R} f|\|_{L^{p}\left(\mathbf{H} H^{n}\right)} \leq c\|f\|_{L^{p}\left(\mathbf{H} H^{n}\right)}, \forall f \in C_{0}^{\infty}\left(\mathbf{H} H^{n}\right) .
$$

\section{References}

[1] D. Allcock, An isoperimetric inequality for the Heisenberg groups, GAFA, Geom. Funct. Anal., 8 (1998), 219-233, MR 99g:53038, Zbl 0907.22009.

[2] S. Barker and S. Salamon, Analysis on a generalized Heisenberg group, J. London Math. Soc., 28 (1983), 184-192, MR 85d:22022, Zbl 0522.22008. 
[3] T. Coulhon, D. Múller and J. Zienkiewicz, About Riesz transforms on the Heisenberg groups, Math. Ann., 305 (1996), 369-379, MR 97f:22015, Zbl 0859.22006.

[4] J. Cygan, Heat kernels for class 2 nilpotent groups, Studia Math., 64 (1979), 227-238, MR 82b:22016, Zbl 0404.35027.

[5] G. Folland, A fundamental solution for a subelliptic operator, Bull. Amer. Math. Soc., 29 (1973), 373-376, MR 47 \#3816, Zbl 0256.35020.

[6] N. Garofalo and F. Segala, Estimates of the fundamental solution and Wiener's criterion for the heat equation on the Heisenberg group, Indiana Univ. Math. Jour., 38(4) (1989), 1155-1196, MR 91k:35138, Zbl 0808.35046.

[7] B. Gaveau, Principle de moindre action, propogation de la chaleur et estimees sous-elliptiques sur certains groups nilpotents, Acta Math., 139 (1997), 95-153, MR 57 \#1574, Zbl 0366.22010.

[8] H. Hueber and D. Müller, Asymptotics for some Green kernels on the Heisenberg group and the Martin boundary, Math. Ann., 283 (1989), 97-119, MR 90b:58257, Zbl 0639.31005.

[9] A. Hulanicki, The distribution of energy of the Brownian motion in Gaussian field and analytic hypoellipticity of certain subelliptic operators on the Heisenberg group, Studia Math., 56 (1976), 165-173, MR 54 \#6298, Zbl 0336.22007.

[10] N. Lohoué and N. Varopoulos, Remarques sur les transformees de Riesz sur les groupes nilpotents, C. R. Acad. Sci. Paris, 301(11) (1985), 559-560, MR 87b:43008, Zbl 0582.43003.

[11] E. Stein, Some results in harmonic analysis in $\mathbf{R}^{n}$ for $n \rightarrow+\infty$, Bull. Amer. Math. Soc., 9 (1983), 71-73, MR 84g:42019, Zbl 0515.42018.

[12] E. Titchmarsh, The Theory of Functions, Oxford University Press, Oxford, 1952, MR 33 \#5850, Zbl 0336.30001.

Received August 9, 2000 and revised June 20, 2002. This work is partially supported by Chinese National Natural Science Foundation Grant-19871063.

INSTITUTE OF MATHEMATiCS

WUHAN UNIVERSITY

WUHAN, 430072

P.R. China

E-mail address: flzhu@whu.edu.cn 